\title{
Repairing Thymic Function
}

Helena Nunes-Cabaço and Ana E. Sousa

Unidade de Imunologia Clínica, Instituto de Medicina Molecular, Faculdade de Medicina, Universidade de Lisboa, Av. Prof. Egas Moniz, 1649-028 Lisboa, Portugal.

\section{Corresponding Author:}

Ana E. Sousa, MD PhD

Unidade de Imunologia Clínica, Instituto de Medicina Molecular, Faculdade de Medicina de Lisboa, Av. Prof. Egas Moniz, 1649-028 Lisboa, Portugal.

Phone: +351217999525 Fax: +351217999527

E-mail: asousa@fm.ul.pt 


\section{Abstract}

Purpose of Review: Maintenance of $\mathrm{T}$ cell function and modulation of tolerance are critical issues in organ transplantation. The thymus is the primary organ for $\mathrm{T}$ cell generation, and a preserved thymic function is essential for a self-tolerant diverse $\mathrm{T}$ cell repertoire. Transplant procedures and related immunosuppressive drugs may hinder thymic integrity and function. We review here recent advances in understanding the regulation of the unique thymic microenvironment with relevance for the field of transplantation.

Recent findings: Recent studies have assigned a role for IL-22 in the regeneration of thymic epithelium, and for micro RNAs in the modulation of its survival and function. The interplay of key molecules in the cross-talk between thymic epithelial cells and thymocytes was depicted, opening new perspectives for the in vitro recapitulation of $\mathrm{T}$ cell development and for thymic transplantation. Additionally, the thymus was shown to be able to sustain thymocyte progenitor renewal.

Summary: These findings open new venues of research towards: 1) Therapeutic interventions in the endogenous thymus to modulate or reconstitute the immune system; 2) Thymic transplantation; and 3) The future development of artificial thymus, which would represent an important tool to achieve tolerance across histocompatibility barriers.

Keywords: Thymus, Thymic transplantation, Thymic regeneration. 


\section{Introduction}

The thymus is the primary organ for $\mathrm{T}$ cell development, where a $\mathrm{T}$ cell repertoire able to recognize foreign antigens, yet tolerant to self, is generated early in life [1]. The contribution of the thymus to T cell homeostasis after the first years of life was neglected for many years due to the acknowledged age-dependent thymic involution [2-4]. However, there is now clear data supporting life-long thymic activity, posing the thymus as a fundamental target for the replenishment of the T cell compartment $[5,6]$. The thymus is sometimes removed in part or whole for cardiac or lung transplantation raising concerns about the life-long impact of thymectomy [7-9]. Organ transplantation is usually associated with the use of immunosuppressive therapies that hinder the thymus. Residual thymic function and thymic regeneration might be key to re-establish host defense [10]. Deliberate thymus manipulation by direct or peripheral injection of antigen or cells might be used to generate allogeneic tolerance [11]. Finally, the thymus itself is sometimes transplanted, either to establish immunity [12] or to generate tolerance [13]. Recent findings set the scene for promising strategies to be used in clinical settings of organ transplantation to ensure immune reconstitution upon immunosuppressive interventions and/or to modulate T cell tolerance (Figure 1).

\section{Thymus transplantation}

Hematopoietic precursors originated in the bone marrow enter the thymus through the corticomedullary junction and must proceed through a series of stages to become mature, functional T cells. These stages are characterized by expression of CD4 and CD8, as well as CD3, at the surface of thymocytes, with the different stages of $T$ cell development being differentially located in the thymus: immature double positive (DP, $C D 4^{+} \mathrm{CD} 8^{+}$) cells are found in the cortex, while more mature $\mathrm{CD} 4$ single positive (CD4SP, $\mathrm{CD} 4^{+} \mathrm{CD} 8^{\text {neg }} \mathrm{CD} 3^{\text {high }}$ ) and $\mathrm{CD} 8$ single positive (CD8SP, $\mathrm{CD} 4^{\text {neg }} \mathrm{CD} 8^{+} \mathrm{CD} 3^{\text {high }}$ ) are located in the medulla [14].

Thymocyte differentiation is dependent on the interaction of developing thymocytes with other cells, particularly thymic epithelial cells (TEC) and dendritic cells (DC), in order to ensure that the $\mathrm{T}$ cells produced are able to recognize self-MHC (positive selection, mainly mediated by cortical TECs) but do not become activated upon self-antigen presentation (negative selection, mediated by DCs and medullary TEC) $[15,16]$. 
Defects in thymic development, like DiGeorge syndrome, or mutations in genes essential for TEC differentiation, such as Forkhead box N1 (FOXN1), may result in athymia, defined by less than $1 \%$ naïve $T$ cells in the blood $[17,18]$. These cases of severe immunodeficiency can only be treated with thymus transplantation. Thymic transplantation, previously applied in the treatment of complete DiGeorge anomaly in infancy [12,19], has been successfully used for the first time in the context of another clinical setting characterized by putative athymia, FOXN1 deficiency $\left[20,21^{\star}\right]$. Importantly, functional immune-competence was obtained using an HLAmismatched thymus, and was sustained despite evidence of allograft involution 3 years post thymus transplantation $\left[21^{\star}\right]$. These findings suggest that a transitory period of thymic activity is sufficient to ensure the establishment of a functional imune system. Furthermore, they provide new insights for the design of immunological reconstitution strategies based on thymic transplantation, including xenotransplantation [22]. Additionally, the potential of the conjoint use of thymus transplantation with hematopoietic stem-cell transplantation (HSCT) to facilitate longterm immune reconstitution in the context of severe combined immunodeficiencies (SCID) is also an area of research [23].

\section{Manipulation of thymic activity in the context of solid organ transplantation}

There is increasing evidence supporting that a normal thymus is able to export mature $T$ cells throughout life, although with a progressive decline with age $[5,6]$. Nevertheless, the current tools available to accurately estimate the diversity and function of the $T$ cells that emigrate from the thymus are limited. Furthermore, the mechanisms underlying thymic changes throughout life, including the relative contribution of putative impairments of precursor cells and of the thymic microenvironment, remain unclear. The progressive decline of thymic function with age is considered an important factor in limiting the success of bone marrow transplantation in the elderly $[24,25]$. Strategies to recover thymic function and to ensure the replenishment of the peripheral compartment with a pool of newly generated diverse T cells are particularly crucial in situations that may lead to $\mathrm{T}$ cell depletion, such as immunosuppressive therapies, radiation/chemotherapy and HIV infection. The thymic epithelium is particularly susceptible to the deleterious effects of stress, inflammation, infections and immunosuppressive drugs, leading to impaired thymopoiesis [26]. Understanding the mechanisms underlying the 
preservation and recovery of the thymic microenvironment is of paramount importance for its therapeutic targeting.

\section{IL-22 as a novel player in thymic epithelium recovery}

Interleukin 22 (IL-22) has been suggested to have a fundamental role in tissue homeostasis and repair [27]. It is produced by subsets of CD4 T cells and of innate lymphoid cells (ILC) and mainly targets epithelial cells. IL-22 was recently identified as an important player in thymic regeneration $\left[28^{* *}, 29\right]$. Dudakov et al. found that IL-22-deficient mice presented impaired thymic regeneration upon sublethal total body irradiation and were unable to reconstitute thymic cellularity upon subsequent hematopoietic stem cell transplantation. Moreover, IL-22 production increased upon thymic insult in wild type mice. IL-22 appeared to be mainly produced by thymic ILCs in response to tissue damage, and this process was at least partly regulated by DCs through the production of IL-23. The receptor for IL-22 was shown to be expressed on TECs and to induce their survival and proliferation. This direct impact of IL-22 on TECs may have many potential applications.

\section{Fine-tuning of thymic epithelium function by micro RNAs}

Thymic involution is mediated by a variety of factors such as sex hormones, physiological stress, inflammation and infection, but the mechanisms underlying the action of each factor are not completely understood. Recently, micro RNAs (miRNAs) have been found to be essential in maintaining the competence of the thymic epithelia in the mouse $\left[30^{\star *}, 31^{* *}\right]$. This class of small noncoding RNAs controls the cellular RNA machinery by targeting and down-regulating the expression of mRNAs [32]. Dicer is an RNAse III critical for miRNA generation by catalyzing miRNA duplex formation [33]. Papadopoulou et al. have found that thymic epithelial cell-specific deletion of Dicer resulted in the degeneration of thymic architecture and function, inducing heightened sensitivity to involution signals $\left[30^{\star *}\right]$. Furthermore, targeted deletion of the miR-29a cluster recapitulated premature thymic involution and hypersensitivity to pathogen-associated signals found in the absence of Dicer, revealing this particular miRNA family as a key modulator of the threshold of thymic epithelium responses to pathogens. 
In another study, Zuklys et al. also found that $T$ cells generated in a miRNA-free TEC environment elicited autoimmunity, possibly due to the reduction of the expression of selfantigens in medullary TECs, leading to an impaired control of auto-reactive T cells in the thymus $\left[31^{* *}\right]$.

Overall, a major role for miRNAs in thymic epithelium function and maintenance was revealed, raising new perspectives for therapeutic intervention.

\section{Identifying the molecular requirements for the cross-talk between TECs and thymocytes}

The identification of the requirements for $\mathrm{T}$ cell commitment and development has been the subject of extensive research. Caldéron and Boehm have recently revealed a hierarchy of molecular cues that governs the differentiation of hematopoietic progenitors in the murine thymus enlagen $\left[34^{\star *}, 35\right]$. In their model, the chemokines Ccl25 and Cxcl12, the cytokine stemcell factor (Scf) and the Notch ligand delta-like 4 (DLL4), not expressed in Foxn1-deficient epithelia, were placed under the regulation of the Foxn1 promoter in Foxn1-deficient mice. Foxn1 is a transcription factor crucial for thymic epithelium development and prevention of its involution [36,37]. While the expression of $\mathrm{Ccl} 5$, Cxcl12 or Scf was sufficient for hematopoietic progenitor cells to be detectable, although at very low levels, DLL4 alone did not induce hematopoietic activity. However, through the combinatorial expression of the four molecules the authors uncovered determinants of the microenvironmental niche required for $\mathrm{T}$ cell development. According to their results, Ccl25 was positioned at the bottom of the functional hierarchy, while DLL4 had a dominant T cell promoting function over the other molecules studied. In the absence of Notch function, Cxcl12 was found to promote B cell development, while Scf appeared to generate a favorable environment for mast cell progenitors. Importantly, the combined expression of Cxcl12 and DLL4 in Foxn1-deficient epithelia was sufficient to support T cell development up to the DP stage, probably due to the chemotactic activity of Cxcl12, lacked by DLL4. Moreover, these DP T cells were able to differentiate into mature CD4SP and CD8SP cells in the presence of wild-type thymic epithelium in reaggregate thymus organ culture, suggesting that interruption of thymopoiesis at that stage was due to the lack of critical extracellular signals in Foxn1-deficient epithelia. 
These results are of paramount importance for the manipulation of hematopoietic progenitor niches, with potential clinical applications.

\section{The human phenotype of FOXN1 deficiency and its recovery by thymic transplantation}

We and others have shown that children with a homozygous FOXN1 mutation (R255X) feature $T$ cells of non-maternal origin, despite the athymia $\left[18,20,21^{*}\right]$. The circulating $T$ cells present an activated memory-like phenotype, impaired function and an oligoclonal repertoire, with an aberrant over-representation of $\mathrm{CD} 4^{\text {neg }} \mathrm{CD} 8^{\text {neg }}$ double negative and of Forkhead box $\mathrm{P} 3$ (FOXP3) regulatory-like $\alpha \beta \mathrm{T}$ cells $\left[21^{*}\right]$. Several possibilities may be raised to explain their origin. A thymic origin of circulating T cells in human FOXN1 deficiency cannot be excluded. It is plausible that the FOXN1 mutation allowed the development of a thymic rudiment able to support altered thymopoiesis, with possible disturbances in positive/negative selection, as suggested by the double negative and FOXP3+ cell expansions. On the other hand, an extrathymic origin should also be considered, although the possibility of $\mathrm{T}$ cell development in lymph nodes and/or gut has been subject of intense debate and controversial data $[38,39]$. In this respect, two recent reports should be considered, which describe the potential of human tonsils to support $\mathrm{T}$ cell development $\left[40^{\star}\right]$ and the possible contribution of mesenteric lymph nodes for extrathymic development of murine $\mathrm{T}$ cells upon administration of bone marrow progenitors $\left[41^{\star}\right]$.

\section{Revealing the thymic ability to sustain thymopoiesis in the absence of progenitor input}

It was long believed that the thymus was entirely dependent on the inner traffic of progenitor cells from the bone marrow. This notion derived from the observation that transplantation of wild type thymus grafts into $\mathrm{T}$ cell deficient mice resulted in the export of a single wave of graftderived $\mathrm{T}$ cells, and that colonization of the graft by developmentally impaired progenitors from the bone marrow resulted in cessation of T cell production $[42,43]$. Peaudecerf et al. $\left[44^{\star \star}\right]$ and Martins et al. $\left[45^{\star *}\right]$ revisited this dogma, showing in mouse models that the thymus harbors progenitors with self-renewal potential that are able to maintain $\mathrm{T}$ cell development in the absence of competition from bone marrow progenitors. These recent studies unveil a 
mechanism of regulation of normal thymocyte development by niche occupancy and competitive progenitor replacement.

Overall, these studies highlight the capacity of the thymus to support cells with long-term renewal potential.

\section{Intrathymic injection of thymocyte progenitors}

The intrathymic delivery of thymocyte progenitors has been investigated as a strategy to reduce the delay in immune reconstitution following hematopoietic stem cell transplantation, a main cause of the associated morbidity and mortality. Recent studies from de Barros et al. in murine models showed that, in the absence of of myeloablative conditioning, the forced thymic entry of semiallogeneic progenitors results in sustained $\mathrm{T}$ cell development, even across histocompatibility barriers $\left[46^{*}\right]$.

\section{Modulating $\mathrm{T}$ cell responses at the thymic level}

Another important area of thymic intervention relates to the role of the thymus in the establishment of the $T$ cell receptor repertoire. Much effort has been devoted into developing ways to achieve hematopoietic chimerism for the purpose of generating central tolerance [11]. Moreover, the development of strategies to induce tolerance through the direct introduction of antigens in the thymus has been a long pursued goal in the field of transplantation $[47,48]$. Several approaches have been attempted to modulate specific $T$ cell responses, including the intrathymic injection of antigens using lentiviral vectors as recently reported by Gottrand et al. $\left[49^{*}\right]$. On the other hand, although the thymus is generally considered an immune-privileged organ, increasing evidence points to a recirculation of mature $T$ cells and antigen-presenting cells through the thymus, with possible modulation of peripheral effector and regulatory $\mathrm{T}$ cell responses [50]. The majority of the studies addressing this issue have been conducted in mouse models of infection, such as the recently published work of Nobrega et al. with Mycobacteria [51*]. This field of research has an immense translational potential in the context of organ transplantation to induce tolerance. In this regard, we have recently identified the DP stage of thymocyte development as a potential target for the modulation of thymic-derived FOXP3 $^{+}$regulatory $\mathrm{T}$ cells $\left[52^{*}\right]$. These findings illustrate an early generation of this important 
subset for T cell homeostasis and self-tolerance, with critical implications for the diversity and autoreactivity of the natural Treg-cell repertoire.

\section{Conclusions}

The thymus has recently emerged as a promissory target for therapeutic intervention in the field of organ transplantation. Its unique regenerative potential and the identification of new key pathways involved raise prospects to modulate or rebuild the patient's immune system. These advances in the comprehension of the thymic microenvironment regulation will be instrumental for therapeutic interventions in the endogenous thymus, thymic transplantation, and future development of artificial thymus, which would represent an important tool to achieve tolerance across histocompatibility barriers.

\section{Key Points:}

1. The central role of the thymus in the establishment of the $T$ cell receptor repertoire and tolerance disclose a potential in organ transplantation that is beginning to be explored.

2. IL-22 was recently revealed as a major pathway underlying the unique ability of the thymus to regenerate.

3. Critical molecular mechanisms that regulate the function of the thymic epithelia were uncovered, which may translate into advances in the modulation of the thymic microenvironment, as well as in the generation of a putative artificial thymus.

4. The thymus was shown to have the ability to sustain thymocyte progenitor renewal.

5. The human phenotype of FOXN1 deficiency, a key molecule for thymic epithelium development and function, and its recovery upon thymic transplantation was comprehensively characterized.

\section{Acknowledgements:}

The authors declare no conflict of interests.

The authors thank Russell Foxall for critical revision of the manuscript. 
Figure Legend

Figure 1: Overview of T cell development in the thymus and recent studies that bring new light to the modulation and boost of thymic function. (DN: double negative, DP: double positive, SP: single positive, CTEC: cortical thymic epithelial cell, mTEC: medullary thymic epithelial cell, DC: dendritic cell). 


\section{References}

1. Miller JFAP. The discovery of thymus function and of thymus-derived lymphocytes. Immunol. Rev. 2002;185:7-14.

2. Simpson JG, Gray ES, Beck JS. Age involution in the normal human adult thymus. Clin. Exp. Immunol. 1975;19(2):261-5.

3. Steinmann GG, Klaus B, Müller-Hermelink HK. The involution of the ageing human thymic epithelium is independent of puberty. A morphometric study. Scand. J. Immunol. 1985;22(5):563-75.

4. Chinn IK, Blackburn CC, Manley NR, Sempowski GD. Changes in primary lymphoid organs with aging. Semin. Immunol. 2012;24(5):309-20.

5. Douek DC, McFarland RD, Keiser PH, et al; Changes in thymic function with age and during the treatment of HIV infection. Nature. 1998;396(6712):690-5.

6. Dion $\mathrm{M}-\mathrm{L}$, Poulin J-F, Bordi $\mathrm{R}$, et al; HIV infection rapidly induces and maintains a substantial suppression of thymocyte proliferation. Immunity. 2004;21(6):757-68.

7. Ogle BM, West LJ, Driscoll DJ, et al; Effacing of the T cell compartment by cardiac transplantation in infancy. J. Immunol. 2006;176(3):1962-7.

8. Platt JL, Cascalho M, West L. Lessons from cardiac transplantation in infancy. Pediatr. Transplant. 2009;13(7):814-9.

9. Sauce D, Appay V. Altered thymic activity in early life: how does it affect the immune system in young adults? Curr. Opin. Immunol. 2011;23(4):543-8.

10. Hollander GA, Krenger W, Blazar BR. Emerging strategies to boost thymic function. Curr. Opin. Pharmacol. 2010;10(4):443-53.

11. Sachs $D H$, Sykes M, Kawai T, Cosimi AB. Immuno-intervention for the induction of transplantation tolerance through mixed chimerism. Semin. Immunol. 2011;23(3):165- 
73.

12. Markert ML, Devlin BH, Mccarthy EA. Thymus transplantation. Clin Immunol. 2010;135(2):236-46.

13. Yamada K, Scalea J. Thymic Transplantation in Pig-to-Nonhuman Primates for the Induction of Tolerance Across Xenogeneic Barriers. Methods Mol. Biol. 2012. 885:191212.

14. Spits $H$. Development of $\alpha \beta T$ cells in the human thymus. Nat Rev Immunol. 2002;2(10):760-72.

15. Starr TK, Jameson SC, Hogquist KA. Positive and Negative Selection of T cells. Annu. Rev. Immunol. 2003;21(1):139-76.

16. Kyewski B, Klein L. A Central Role for Central Tolerance. Annu. Rev. Immunol. 2006;24(1):571-606.

17. Frank J, Pignata C, Panteleyev AA, et al; Exposing the human nude phenotype. Nature. 1999;398(6727):473-4.

18. Romano R, Palamaro L, Fusco A, lannace L, Maio S, Vigliano I, et al. From murine to human nude/SCID: the thymus, T-cell development and the missing link. Clin. Dev. Immunol. 2012;2012:467101.

19. Markert ML, Devlin BH, Chinn IK, et al; Factors Affecting Success of Thymus Transplantation for Complete DiGeorge Anomaly. Am. J. Transplant. 2008;8(8):1729-36.

20. Markert ML, Marques JG, Neven B, et al; First use of thymus transplantation therapy for FOXN1 deficiency (nude/SCID): a report of 2 cases. Blood. 2011;117(2):688-96.

*21. Albuquerque AS, Marques JG, Silva SL, et al; Human FOXN1-Deficiency Is Associated with $\alpha \beta$ Double-Negative and FoxP3+ T-Cell Expansions That Are Distinctly Modulated upon Thymic Transplantation. PLoS ONE. 2012;7(5):e37042.

This work reveals the presence of circulating $T$ cells in the context of an homozygous 
mutation in human FOXN1 and the achievement of long-term functional immunecompetence upon HLA-mismatched thymic transplantation.

22. Zhou J, Wang X, Luo G, et al; Partial Tolerance Induced by Transplantation of Spatially Separated Thymuses: A Cue for T Cell Retolerization in Thymus Grafts. Scand J Immunol. 2012;75(4):401-8.

23. Hu Z, Yang Y-G. Human lymphohematopoietic reconstitution and immune function in immunodeficient mice receiving cotransplantation of human thymic tissue and CD34(+) cells. Cell. Mol. Immunol. 2012;9(3):232-6.

24. Hakim FT, Memon SA, Cepeda R, et al. Age-dependent incidence, time course, and consequences of thymic renewal in adults. J. Clin. Invest. 2005;115(4):930-9.

25. Krenger W, Blazar BR, Hollander GA. Thymic T-cell development in allogeneic stem cell transplantation. Blood. 2011;117(25):6768-76.

26. Pujol-Borrell R, Herrero-Mata MJ, Palou E, Armengol MP. Immunological senescence and thymic function in transplantation. Transplantation. 2009;88(3 Suppl):S8-13.

27. Sonnenberg GF, Fouser LA, Artis D. Border patrol: regulation of immunity, inflammation and tissue homeostasis at barrier surfaces by IL-22. Nat Immunol. 2011;12(5):383-90.

**28. Dudakov JA, Hanash AM, Jenq RR, et al; Interleukin-22 Drives Endogenous Thymic Regeneration in Mice. Science. 2012;336(6077):91-5.

This work unveils a mechanism of endogenous murine thymic regeneration that relies on IL-22. The authors describe a population of radio-resistant innate-like lymphocytes (ILCS) that produce IL-22 upon thymic insult in an IL-23-dependent manner, leading to the survival and proliferation of TECs. Thymic recovery upon IL-22 administration after irradiation reveals a possible novel therapeutic target for the regeneration of thymic function.

29. Bhandoola A, Artis D. Immunology. Rebuilding the thymus. Science. 2012;336(6077):40-1. 
**30. Papadopoulou AS, Dooley J, Linterman MA, et al; The thymic epithelial microRNA network elevates the threshold for infection-associated thymic involution via miR-29a mediated suppression of the IFN- $\alpha$ receptor. Nat Immunol. 2012;13(2):181-7.

This work reveals a link between the thymic epithelial miRNA network and thymic involution, specifically in the context of infection, in the mouse. The authors show that when the thymic epithelium is deficient for the endoribonuclease Dicer, critical for miRNA formation, or in the miR-29a cluster, suboptimal signals are sufficient to trigger a rapid loss of thymic cellularity due to an increased sensitivity to infection signals.

**31. Zuklys S, Mayer CE, Zhanybekova S, et al; MicroRNAs Control the Maintenance of Thymic Epithelia and Their Competence for T Lineage Commitment and Thymocyte Selection. J. Immunol. 2012;189(8):3894-904.

In this study the authors found that mice in which the thymic epithelium lacks the endoribonuclease Dicer, critical for miRNA formation, have reduced positive thymocyte selection and an altered $T$ cell repertoire. Furthermore, $T$ cells generated in a miRNAfree TEC environment elicited autoimmunity, as observed by multiorgan infiltration.

32. Bartel DP. MicroRNAs: target recognition and regulatory functions. Cell. 2009;136(2):215-33.

33. Kim VN. MicroRNA biogenesis: coordinated cropping and dicing. Nat. Rev. Mol. Cell Biol. 2005;6(5):376-85.

**34. Calderón L, Boehm T. Synergistic, Context-Dependent, and Hierarchical Functions of Epithelial Components in Thymic Microenvironments. Cell. 2012;149(1):159-72.

In this work important molecular cues governing the murine thymocyte progenitor niche are deciphered. The individual and combinatorial expression on thymic epithelial cells of the chemokines CXCL12 and CCL25, of the Notch ligand delta-like 4 (DLL4) and of the cytokine stem-cell factor Scf allowed the division of the hierarchical interactions that regulate the thymocyte progenitor niche. 
35. Krause DS, Scadden DT. Deconstructing the Complexity of a Microenvironmental Niche. Cell. 2012;149(1):16-7.

36. Chen L, Xiao S, Manley NR. Foxn1 is required to maintain the postnatal thymic microenvironment in a dosage-sensitive manner. Blood. 2009;113(3):567-74.

37. Corbeaux T, Hess I, Swann JB, et al; Thymopoiesis in mice depends on a Foxn1positive thymic epithelial cell lineage. Proc Natl Acad Sci. 2010;107(38):16613-8.

38. Rocha B. The extrathymic T-cell differentiation in the murine gut. Immunol. Rev. 2007;215:166-77.

39. Blais M-E, Brochu S, Giroux M, et al; Why T cells of thymic versus extrathymic origin are functionally different. J. Immunol. 2008;180(4):2299-312.

*40. McClory S, Hughes T, Freud AG, et al; Evidence for a stepwise program of extrathymic T cell development within the human tonsil. J. Clin. Invest. 2012;122(4):1403-15. In this work the authors report a program of T cell development that occurs in the human tonsil.

*41. Holland AM, Zakrzewski JL, Tsai JJ, et al; Extrathymic development of murine T cells after bone marrow transplantation. J. Clin. Invest. 2012;122(12):4716-26.

This study supports the occurrence of extrathymic $T$ cell develoment in mesenteric lymph nodes in murine models.

42. Frey JR, Ernst B, Surh CD, Sprent J. Thymus-grafted SCID mice show transient thymopoiesis and limited depletion of $\mathrm{V}$ beta $11+\mathrm{T}$ cells. J. Exp. Med. 2012;175(4):1067-71.

43. Takeda $\mathrm{S}$, Rodewald $\mathrm{H}-\mathrm{R}$, Arakawa $\mathrm{H}$, et al; $\mathrm{MHC}$ class II molecules are not required for survival of newly generated CD4+ T cells, but affect their long-term life span. Immunity. $1996 ; 5(3): 217-28$.

**44. Peaudecerf L, Lemos S, Galgano A, et al; Thymocytes may persist and differentiate without any input from bone marrow progenitors. J. Exp. Med. 2012;209(8):1397-400. 
This work challenges the dogma that thymocyte progenitors do not have the ability to self-renew in the thymus. The authors show that the murine newborn thymus contains progenitors that are able to maintain $T$ cell differentiation and export for several months and to ensure partial or total protection to infection. An essential role for competition for IL-7 between incoming and resident progenitor cells is described.

**45. Martins VC, Ruggiero E, Schlenner SM, et al; Thymus-autonomous T cell development in the absence of progenitor import. J. Exp. Med. 2012;209(8):1409-17.

This work published in the same issue as Peaudecerf et al. also support an ablity of thymocyte progenitors to self-renew in the thymus. The authors show that the murine newborn thymus contains progenitors that are able to maintain $T$ cell differentiation and export for several months, and that $\gamma_{c}$-mediated signals play a pivotal role in the competition for the niche between thymic resident and recently ingressed progenitors.

*46. de Barros SC, Vicente R, Chebli K, et al; Intrathymic progenitor cell transplantation across histocompatibility barriers results in the persistence of early thymic progenitors and T cell differentiation. Blood. 2013; in press.

The authors show that, in the absence of myeloablative conditioning, intrathymic (but not intravenous) injection of semiallogeneic hematopoietic stem cells resulted in sustained $T$ cell development, supporting the long-term renewal potential of the thymus.

47. Ellison GW, Waksman BH. Role of the thymus in tolerance. IX. Inhibition of experimental autoallergic encephalomyelitis by intrathymic injection of encephalitogen. J. Immunol. 1970;105(2):322-6.

48. Posselt AM, Barker CF, Tomaszewski JE, et al; Induction of donor-specific unresponsiveness by intrathymic islet transplantation. Science. 1990;249(4974):1293-5.

*49. Gottrand G, Taleb K, Ragon I, et al; Intrathymic injection of lentiviral vector curtails the immune response in the periphery of normal mice. J. Gene Med. 2012;14(2):90-9.

In this study the authors show that intrathymic injection of a lentiviral vector induces peripheral tolerance to the same antigen. The intrathymic route is found to be a promising venue for gene transfer in the modulation of $T$ cell tolerance and 
autoimmunity.

50. Hale JS, Fink PJ. Back to the thymus: peripheral T cells come home. Immunol. Cell Biol. 2008;87(1):58-64.

*51. Nobrega C, Nunes-Alves C, Cerqueira-Rodrigues B, et al; T Cells Home to the Thymus and Control Infection. J. Immunol. 2013; 190(4):1646-58.

In this work Nobrega et al. describe the presence of recirculating mycobacteria-specific $C D 4+$ and $C D 8+T$ cells in the thymus following dissemination of Mycobacterium avium or Mycobacterium tuberculosis. The data support $T$ cell recirculation from the peripehry into the thymus as a mechanism of controling thymic infection.

*52. Nunes-Cabaço H, Caramalho I, Sepúlveda N, Sousa AE. Differentiation of human thymic regulatory T cells at the double positive stage. Eur. J. Immunol. 2011;41(12):3604-14. This study identifies the early double positive stage of thymocyte development as a potential target for the modulation of thymic-derived FOXP3 $3^{+}$regulatory $T$ cells. 